\title{
Successful Rituximab Treatment for Lymphoma, Secondary Immunodeficiency Causing Debilitating Sinusitis: Underlying Primary Immunodeficiency Disease, and Alternative Treatments to Improve the Quality of Life?
}

\author{
Vincent R. Bonagura ${ }^{1}$ (i) \\ Received: 22 April 2019 / Accepted: 22 April 2019 / Published online: 17 May 2019 \\ (C) Springer Science+Business Media, LLC, part of Springer Nature 2019
}

The Letter to the Editor by Dr. Palmeira et al. in this issue of the Journal of Clinical Immunology brings up several challenges for clinical immunologists who care for patients with secondary immunodeficiency disease caused by modern aggressive chemotherapy or biologics that are used to treat autoimmune and malignant diseases. The authors describe a case of recalcitrant sinusitis in a 76-year-old woman who was treated for lymphoma with several cycles of rituximab. The "punchline" of this Letter is that alternative treatments, other than IgG replacement therapy (IGRT) and chronic antibiotics, need to be developed to treat recalcitrant chronic sinusitis that causes poor quality of life patients after successful treatment of their autoimmune disease or cancer with medications that cause delayed or complete failure to reconstitute competent mucosal immunity.

There are three challenges inherent in this Letter. First, secondary immunodeficiency occurs in some patients treated for autoimmune disease or cancer by modern aggressive chemotherapy/biologics, especially rituximab. These drugs can cause a failure to restore adaptive immunity once the underlying disease has successfully been treated. They develop chronic sinorespiratory disease that is not responsive to chronic antibiotics and/or IGRT. Second, although not addressed in this case report, some of the patients who fail to recover immunologically from these treatments have an underlying primary immune deficiency disease (PIDD). Third, and addressed in this Letter, the development of alternative strategies is needed to treat such patients who develop poorly controlled

Vincent R. Bonagura

VBonagura@northwell.edu

1 Chief, Division of Allergy/Immunology, Department of Pediatrics, Donald and Barbara Zucker School of Medicine at Hofstra/ Northwell, Great Neck, New York, USA sinorespiratory disease. Such protocols may greatly improve the quality of life of these patients.

There is an increasing incidence of drug induced, secondary immunodeficiency conditions $[1,2]$, in a sizable number of patients being treated for autoimmune disease or cancer, using various chemotherapeutic drugs [3] and in particular, rituximab [1-3]. These patients fail to recover adaptive immunity, mostly B cell function, long after successful chemotherapeutic or immune modulator therapy has been discontinued $[1,2]$. Rituximab has been successfully used in treating many diseases that were previously refractory to chemotherapeutic protocols alone. On occasion, rituximab can cause persistent $\mathrm{B}$ cell failure that persists many years after completion of this therapy when patients are in remission. In some patients, there is a significant or complete failure of B cell reconstitution, with poor expression of protective serum immunoglobulin levels to multiple pathogens greater than 8 years following the discontinuation among of rituximab [1].

While we were among the first to report this phenomenon [1], others have confirmed these findings [3]. At the time of our report, we had not yet completed an in-depth immunologic evaluation of the original 14 patients we reported who failed to reconstitute B cell numbers and/or function. Two of these patients after immunologic evaluation had underlying primary immunodeficiency diseases (PIDD), previously unrecognized by the referring physicians who treated them with rituximab and other chemotherapeutic drugs for autoimmune disease or cancer. Thus, the referring physicians had not an not suspected PIDD as a possible underlying cause of the autoimmune disease/cancer their patients had developed prior to giving them these drugs.

The first patient presented with autoimmune thrombocytopenia (Evan syndrome) and we identified a gain of function, PI3Kinase defect in this patient by whole exon sequencing. Unfortunately, no immunophenotype was performed prior to 
instituting rituximab therapy, but on review of endoscopy biopsies performed for severe colitis, B cells were present in these tissues prior to rituximab treatment. He continues on IGRT post rituximab treatment and consistently shows $0-2$ $\mathrm{B}$ cells $/ \mathrm{mL}$ in his blood 8 years after discontinuation of rituximab. He is now treated with sirolimus, but continues to have chronic sinusitis, reactive airway disease, and ground glass opacities in both lungs. His chronic sinusitis has reduced his quality of life, as described in the current patient in the Letter to the Editor in this issue of the Journal, and he has undergone two sinus surgeries that partially controlled his sinus disease. His chronic sinusitis is poorly responsive to chronic antibiotics and IGRT.

The second patient had a diagnosis of autoimmune sinusitis, lung disease, and GI disease and was maintained on moderate doses of steroids, chemotherapy, rituximab, IGRT, and chronic antibiotics. She had abnormal serial immune phenotypes, including low B and $\mathrm{T}$ cell numbers and poor function that were attributed to steroid, rituximab, and chemotherapy treatment. On SCID genetic screening, she was shown to have Rag1 deficiency 5 years after presentation of her symptoms. This patient received an allogeneic stem cell transplant and is now stable on IGRT and antibiotics for chronic sinus and respiratory disease, but her sinus disease is poorly controlled on this regimen. Thus, clinicians should anticipate the possible development of long-term secondary immunodeficiency disease in their patients successfully treated with modern aggressive chemotherapy/biologics, including, but not limited to rituximab, and as part of their follow up strategy be vigilant in instituting appropriate medical management when this condition develops. [2]. Furthermore, clinicians should have a high suspicion in their patients who fail to resolve secondary immunodeficiency long after discontinuation of these drugs, that they may have an underlying PIDD. Thus, an in-depth immune evaluation, including whole exon sequencing, should be considered by clinicians caring for such patients.

Last, as described in this Letter to the Editor, alternative strategies need to be developed to address chronic sinorespiratory disease in patients with secondary immunodeficiency who are in remission, long after immunosuppressive therapy has been discontinued. Reports of topical therapies using IgG immunoglobulin given nasally, or as eye drops, and non-cellular extracts of breast milk, as described in the current Letter to the Editor, are anecdotal. However, a systematic study of the use of these interventions in large numbers of patients has not been conducted. Such strategies used to treat patients with chronic sinorespiratory disease who fail to respond to chronic antibiotics and IGRT need to be developed.

While the authors of the Letter infer that secretory IgA in the supernatant of breast milk is the reason why their patient improved her chronic sinusitis disease, breast milk also contains many anti-inflammatory molecules that can block inflammation and support bacterial clearance [4]. Thus, it is unclear why the therapy reported in this Letter was effective in controlling this patient's chronic sinusitis. Of note, the method used to purify serum IgG for intravenous/ subcutaneous infusions in patients with primary and secondary immunodeficiency syndromes discards dimeric serum IgA as a by-product. This dimeric IgA could be available for clinical use in such patients, and in others with chronic sinusitis who do not have primary and secondary immunodeficiency syndromes. It would be of interest to know if aerosolized serum IgA from the blood of health individuals can be successfully used to treat sinorespiratory disease in these patients to improve their quality of life. An alternative approach might be the use of some of the immunosuppressive molecules present in the non-cellular fraction of human breast milk [3] in clinical trials to determine if these molecules improve the quality of life of patients with persistent sinorespiratory infections refractory to chronic antibiotics and IGRT.

Vincent R. Bonagura

Co-Editor in Chief, Journal of Clinical Immunology

\section{References}

1. Kaplan B, Kopyltsova Y, Khokhar A, Lam F, Bonagura V. Rituximab and immune deficiency: case series and review of the literature. J Allergy Clin Immunol Pract. 2014;2:594-600.

2. Kaplan B, Bonagura VR. Secondary hypogammaglobulinemia an increasingly recognized complication of treatment with immunomodulators and after solid organ transplantation. Immunol Allergy Clin North Am. 2019;39:31-9 Kobrynski, L Ed.

3. AU Casulo C, Maragulia J, Zelenetz AD. Incidence of hypogammaglobulinemia in patients receiving rituximab and the use of intravenous immunoglobulin for recurrent infections. Clin Lymphoma Myeloma Leuk. 2013;2013(13):106-11.

4. Arnardottir H, Orr SK, Dalli J, Serhan CN. Human milk proresolving mediators stimulate resolution of acute inflammation. Mucosal Immunol. 2016;9:757-66.

Publisher's Note Springer Nature remains neutral with regard to jurisdictional claims in published maps and institutional affiliations. 